\title{
Protective Effect of Caffeic Acid on Streptozotocin Induced Gestational Diabetes Mellitus in Rats: Possible Mechanism
}

\author{
Ying Liu ${ }^{1}$, Shankun Liu', Hui Wang ${ }^{2}$ and Weihua $\mathrm{Su}^{2, *}$ \\ ${ }^{1}$ Department of Gynecology, Taian City Central Hospital, No. 29, Longtan Road, \\ Taian, Shandong Province, 271000, China \\ ${ }^{2}$ Departmrnt of Obstetrics, Taian City Central Hospital, No. 29, Longtan Road, \\ Taian, Shandong Province, 271000, China
}

\begin{abstract}
A B S T RA C T
Gestational diabetes mellitus (GDM) is an atypical condition of pregnancy outcome that affect the maternity and infant. Pregnant women are a high risk to develop the GDM suffering from the diabetes, it showed the effect on the development and growth of the fetus. During the GDM increase the incidence of oxidative stress. Caffeic acid is a powerful free radical scavenger and possesses antioxidant effect against the various diseases. Previous study suggests that the caffeic acid have potent anti-inflammatory activity. The aim of the current study was to scrutinize the protective effect of caffeic acid against the streptozotocin induced GDM in rats and explores the possible mechanism of action. A total 36 female rats were caged into the male rats for the pregnancy, and 33 female pregnant rats were collected and weight. The female rats were group into following groups and each group contains 6 rats. Once the pregnancy was verified, the streptozotocin (STZ) single intraperitoneal injection was used for the induction of diabetes. The rats were received the various dose of caffeic acid. After the pregnancy (19 day) the blood sample was collected via puncturing the retro arbital and collecting the fetus from all the groups. The rats, fetus weight and placental weight were calculated. The blood glucose level, serum, lipids, antioxidant, cytokines and C-peptide was recorded. GDM group rats showed the lower weight of pregnant rats, fetal and placental weight as compared to other group rats. Caffeic acid treated group rats exhibited the modulated level of blood glucose level, lipid profile and antioxidant parameters at dose dependent manner. Caffeic acid significantly $(\mathrm{P}<0.001)$ enhanced the level of insulin, hepatic glycogen as compared to GDM control group rats. Caffeic acid significantly $(\mathrm{P}<0.001)$ increased the level of antioxidant enzymes in the liver and pancreas tissue at dose dependent manner. Our finding clearly supported the beneficial effect of caffeic acid on not only the diabetes mellitus, but also alleviating Gestational diabetes mellitus.
\end{abstract}

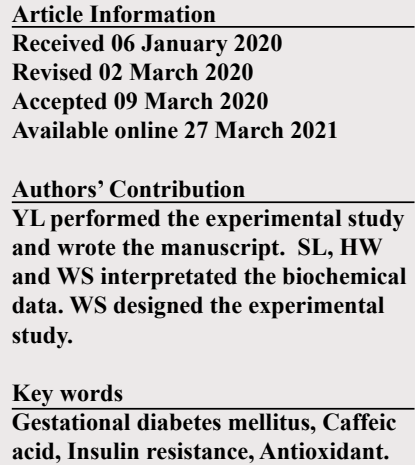

\section{INTRODUCTION}

$\mathrm{C}$ linical studies suggest that the diabetes affects the $>5 \%$ of pregnant women during the pregnancy, inducing the abnormalities in the reproductive that enhance the risk of congenital anomalies, intrauterine growth retardation, spontaneous abortion, mortality and morbidity of both newborn/fetus and mother (Wang et al., 2019). Gestational diabetes mellitus (GDM) is a typical condition during the pregnancy due to insufficient production of insulin. GDM seriously spoils the maternal and infant health (Alfadhli, 2015). Previous studies suggest that the patient suffering from the GDM usually did not show any sign and symptom of diabetes erstwhile to pregnancy, and frequently detected during the $2^{\text {nd }}$ trimester of pregnancy, with $3-5 \%$ of women even suffering long term diabetes mellitus after the

\footnotetext{
Corresponding author: sdtasuweihua@sina.com 0030-9923/2021/0003-1045 \$ 9.00/0

Copyright 2021 Zoological Society of Pakistan
}

pregnancy (Coustan, 2013; Lefkovits et al., 2019). During the expansion of GDM during the maternal period, showed the effect on the fetal and exhibited the development of abnormal fetal. Even though hyperglycaemia is major factor, take part in the pathogenesis of diabetic embryopathy, and increase the incidence of malformations (Bain et al., 2015; Maresh, 2005). It is well proofed that

\footnotetext{
Abbreviations

GDM, gestational diabetes mellitus; STZ, streptozotocin; CA, caffeic acid; P38 MAPK, p38 mitogen-activated protein kinases; EDTA, etheylenediamine tetra acetic acid; DTNB, 5,5 dithiobis-2nitrobenzoic acid; MOPS, morpholinopropansulfonic acid; HEPES, 4,2 hydroxyethyl-1-piperazineethanesulfonic acid; EGTA, ethyleneglycolbis (2-aminoethylether) -N,N,Ń,Ń- tetraacetic acid; DCFH-DA, $2^{\prime} 7^{\prime}$ - dicholorofluoresceindiacetate; TBA, thiobarbutiric acid; TCA, trichloroacetic acid; DNPH, 2,4 dinitrophenylhydrazine; PBS, phosphate buffer saline; HbA1c, glycosylated haemoglobin; CP, C-peptide; TG, triglyceride; TC, total cholesterol; HDL, high density lipoprotein; LDL, low density lipoprotein; VLDL, very low-density lipoprotein; SOD, superoxide mutase; GSH, glutathione; CAT, catalase; PC, protein carbonyl; MDA, malonaldehyde; GPx, glutathione peroxidise; and LPO, lipid peroxidation.
} 
the incidence of fetopathy and embryopathy increased due to oxidative stress and during the diabetes and other metabolic disturbance induced the generation of oxidative stress (Maritim et al., 2003; Spaight et al., 2016). The most protuberant anti-teratogenic effect was accomplished via anti-oxidant therapy, it's not decrease the incidence the teratogenic, also control the glycaemic control, this is beneficial for both outset and during pregnancy (Hong et al., 2017; Yao et al., 2015). Various published research suggests that the supplementation with transgenic of $\mathrm{Cu}$ / $\mathrm{Zn}$ superoxide dismutase or antioxidants such as vitamin $\mathrm{C}$ and vitamin $\mathrm{E}$ could be valuable in improvement of diabetes linked teratogenic effect and oxidative stress. Several researchers make attempt to use the natural phytoconstituent in mitigation of oxidative stress and thus decrease the embryopathy under the diabetic conditions (Hong et al., 2017; Yao et al., 2015; Zou et al., 2018).

Caffeic acid (CA), 3,4-dihydroxycinnamic acida natural phenolic phyto-constituent widely present in the several medicinal plants such as vegetable, fruits, olive oil, wine and also present in the human plasma in various concentration depend upon the diet (Peyrat-Maillard et al., 2003; Sato et al., 2011). Its synthetic derivative also available and posses multiple activity including anticancer, anti-inflammatory, antifibrosis, antihypertensive, metal chelating agent, antioxidant, antiviral, antidepressive, antidiabetic and antibacterial (de Souza and Spinelli, 2009; Sul et al., 2009). CA also reduces the multiple inflammatory pathways against the various cell lines due to its anti-inflammatory nature. It also suppress the LPS induced inflammation against the Raw 264.7 cells via inhibition of p38 mitogen-activated protein kinases (p38MAPK), NH-2 terminal kinase $1 / 2$ activation, nuclear factor Kappa B (NF-kB) (Chen and Ho, 1997; Rajendra Prasad et al., 2011). However, its fetal development using rat model of gestational diabetes mellitus remain unclear. Hence, the aim of the current experimental investigation to scrutinize the fetal protective effect of caffeic acid on GDM induced rats and explores the possible mechanism of action.

\section{MATERIALS AND METHODS}

\section{Chemicals}

Streptozotocin and caffeic acid was purchased from the Sigma Chemical Co. U.S.A. Comassie blue, sodium citrate, etheylenediamine tetra acetic acid (EDTA), glutation, 5,5 dithiobis-2-nitrobenzoic acid (DTNB), tris-Hcl, morpholinopropansulfonic acid (MOPS), 4,2 hydroxyethyl-1-piperazineethanesulfonic acid (HEPES), ethyleneglycol- bis (2-aminoethylether) -N,N,Ń,Ń- tetraacetic acid (EGTA), magnesium chloride
$\left(\mathrm{MgCl}_{2}\right)$, hydrochloride (HCL), potassium chloride $(\mathrm{KCl})$, sodium hydroxide $(\mathrm{NaOH})$, n-butanol, 2' $7^{\prime}$ dicholorofluoresceindiacetate (DCFH-DA), ethylacetate, thiobarbutiric acid (TBA), trichloroacetic acid (TCA), phosphoric acid, 2,4 dinitrophenylhydrazine (DNPH) and guanine hydrochloride.

\section{Experimental animals}

Female and male Wistar rat weight 150-180 g 10-12 old used for the current investigation study. The Wistar rats were received from the animal house and kept in the standard condition $\left(20 \pm 5^{\circ} \mathrm{C}\right.$ temperature; $60-80 \%$ relative humidity; $12 \mathrm{~h}$ light and $12 \mathrm{~h}$ dark cycle). The rats were received the standard pellet chow and water ab libitum. All the experimental protocol performed according to the protocol approved by the University animal ethical committee.

\section{Experimental study}

Briefly, the weight of the experimental rats was maintained between $240-280 \mathrm{~g}$ at 12 weeks. The rats having the blood glucose level (BGL) more than the 6.8 $\mathrm{mmol} / \mathrm{L}$ excluded from the current experimental study. Both male and female rats were allowed to mate and female deficient pessary next day was considered pregnant for 0.5 days. Intravenously (tail vein) administration of streptozotocin (STZ) $25 \mathrm{mg} / \mathrm{kg}$ (dissolve in the citrate buffer, $\mathrm{pH}=4.5$ ) was used for induce GDM and untreated pregnant female rats were used as normal control in the current experimental study (Wang et al., 2019).

After induction of STZ administration, the blood was collected from the retro orbital and blood glucose was estimated at regular time interval via using the glucose oxidase method with minor modification. After successfully induction the diabetes, the female rats were anesthetized and embryos were excised on the ice and some embryos was homogenized in the phosphate buffer saline (PBS) and centrifuged at $8000 \mathrm{rpm}$ at $4^{\circ} \mathrm{C}$ for 10 min and stored in the deep refrigerator at $-20^{\circ} \mathrm{C}$.

\section{Scrutinize the fetal development}

For the estimation of fetal development, the plasma insulin, blood glucose level, plasma insulin, RAGE and AGEs level in the different tissue of the pregnant rats were determine at regular time interval via using ELISA kits following the manufacture instruction. At end of the experimental protocol, the placenta and foetuses were successfully separated and the weight and number of placenta and foetuses were recorded. Finally, the microscopical evaluation was performed to determine the malformations and defects of each organ of fetal (Tang et al., 2014; Wang et al., 2019). 


\section{Biochemical parameters}

Glycosylated haemoglobin Alc (HbAlc), serum C-peptide (CP), hexokinase, hepatic glycogen, glucose6-phosphatase, fructose-1,6 biphosphatase (Kumar et al., 2013a, b) and free fatty acid (FFA) were estimated using ELISA kit following the manufacturer's instruction.

\section{Lipid parameters}

Lipid parameters such as triglyceride (TG), total cholesterol (TC), high density lipoprotein (HDL), low density lipoprotein (LDL) and very low-density lipoprotein (VLDL) were estimated using colorimetric kits following the manufacturer's instruction (Abcam).

\section{Antioxidant parameters}

Antioxidant parameters such as superoxide mutase (SOD), glutathione (GSH), catalase (CAT), protein carbonyl (PC), malonaldehyde (MDA) and glutathione peroxidase (GPx) were estimated via using the previous method with minor modification in the hepatic and pancreas tissue (Shivananjappa and Muralidhara, 2012).

\section{Statistics analysis}

One-way ANOVA was used for the multiple comparisons. Graph pad prism was used for statistical analysis. $\mathrm{P}<0.05$ was considered as statistically significant.

\section{RESULTS}

\section{Effect of caffeic acid on blood glucose}

Figure 1A demonstrated the effect of caffeic acid on STZ induced GDM rats. Normal control group rats showed the almost similar trend in the blood glucose pattern till end of the experimental study. STZ induced GDM group rats showed the increased blood glucose level and increased blood glucose level remain increased till end of the experimental study. STZ induced GDM group rats treated with the caffeic acid significantly $(\mathrm{P}<0.001)$ downregulated as compared to the STZ induced GDM rats.

\section{Effect of caffeic acid on body weight}

Body weight of all group rats presented in Figure 1B. Figure $1 \mathrm{~B}$ exhibited body weight of rats before and after the pregnancy. Normal group pregnant rats demonstrated the increased body weight before and after the pregnancy. STZ induced GDM group rats showed the decreased body weight as compared to normal control rats. Dose dependently treatment of caffeic acid significantly $(\mathrm{P}<0.001)$ increased the body weight as compared to STZ induced GDM rats.

\section{Effect of caffeic acid on AGEs level}

The AGEs level of normal control group rats remains unchanged till complete experimental protocol. On the country, STZ induced GDM rats exhibited the increased level of AGEs as comparison to normal control group rats. STZ induced GDM rats showed reduced level of AGEs at dose dependent manner (Fig. 1C).

\section{Effect of caffeic acid on biochemical parameters}

Figure 2 exhibited the effect of caffeic acid on $\mathrm{HbA} 1 \mathrm{c}$, serum C-peptide, free fatty acid, FINS and hepatic glycogen. Normal control group rats showed the almost normal level of biochemical parameters. STZ induced GDM rats showed the increased level of HbAlc, serum C-peptide, free fatty acid, FINS and dose dependent manner of caffeic acid showed the reduction the level of HbA1c, serum C-peptide, free fatty acid and FINS (Fig. 2).

An opposite trend was observed in the level of hepatic glycogen in the STZ induced GDM rats and dose dependently treatment of caffeic acid significantly $(\mathrm{P}<0.001)$ enhanced the level of hepatic glycogen.
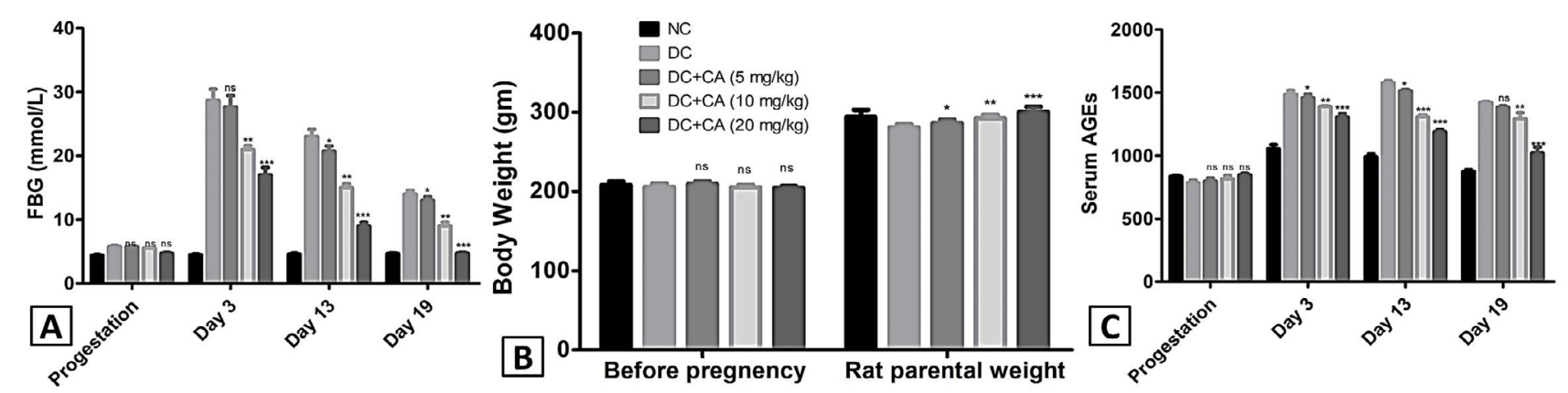

Fig. 1. The effect of caffeic acid on the blood glucose level (A), body weight (B) and serum AGEs (C) of streptozotocin (STZ) induced gestational diabetes mellitus (GDM) induced rats at regular time intervals. Dunnett's multiple test was performed for the statistical analysis. Treated group rats compared with the STZ induced GDM group rats. ${ }^{*} \mathrm{p}<0.05$ consider as the significant, ${ }^{* *} \mathrm{p}<0.01$ consider as the more significant and ${ }^{* * *} \mathrm{p}<0.001$ consider as the most significant. 

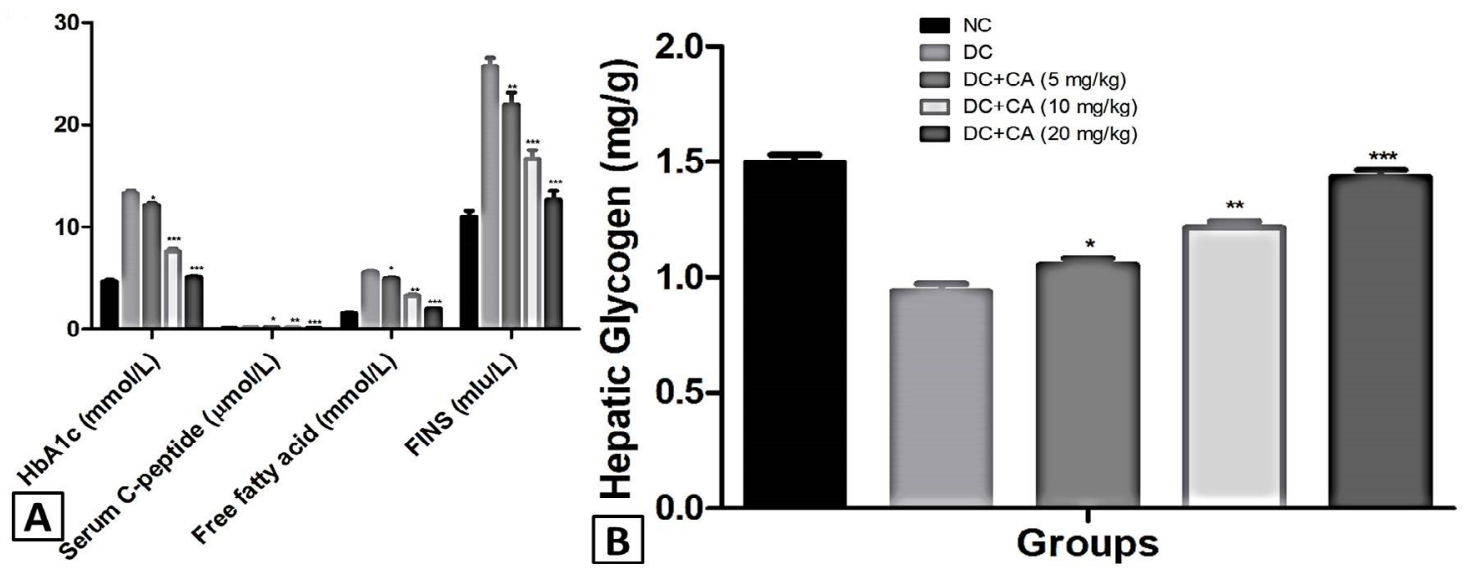

Fig. 2. The effect of caffeic acid on the biochemical parameter of streptozotocin (STZ) induced gestational diabetes mellitus (GDM) induced rats at end of the experimental study. A, the HbA1c, serum C-peptide, free fatty acid, FINSand; B, hepatic glycogen. Dunnett's multiple test was performed for the statistical analysis. Treated group rats compared with the STZ induced GDM group rats. ${ }^{*} \mathrm{p}<0.05$ consider as the significant, ${ }^{* *} \mathrm{p}<0.01$ consider as the more significant and ${ }^{* * *} \mathrm{p}<0.001$ consider as the most significant.

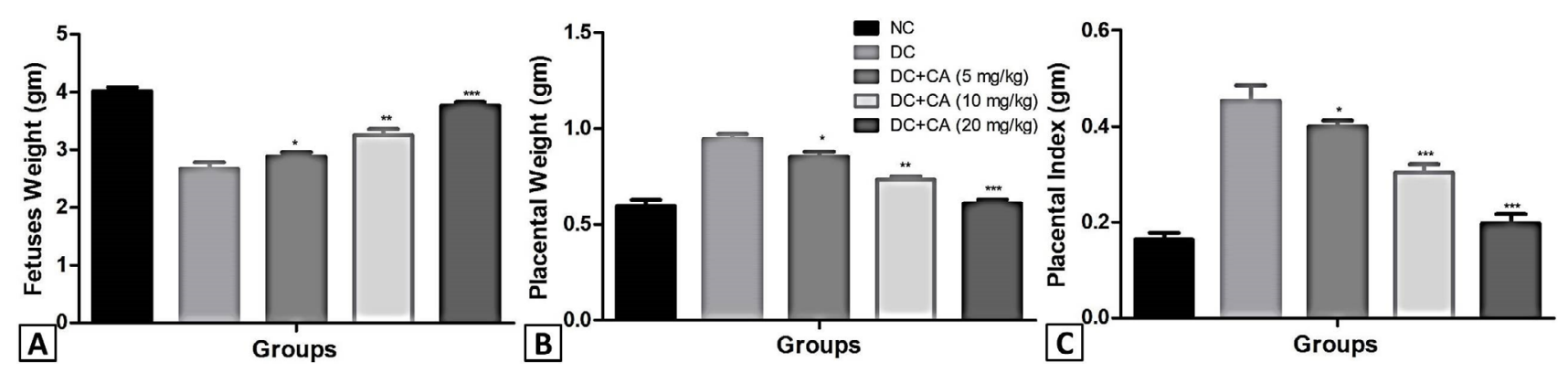

Fig. 3. The effect of caffeic acid on the fetus weight, placental weight and placental index of streptozotocin (STZ) induced gestational diabetes mellitus (GDM) induced rats at end of the experimental study. A, fetuses weight; B, placental weight; C, placental index. Dunnett's multiple test was performed for the statistical analysis. Treated group rats compared with the STZ induced GDM group rats. ${ }^{*} \mathrm{p}<0.05$ consider as the significant, ${ }^{*} \mathrm{p}<0.01$ consider as the more significant and $* * * \mathrm{p}<0.001$ consider as the most significant.

\section{Effect of caffeic acid on fetuses}

Figure 3 illustrated the effect of caffeic acid on the fetuses weight, placental weight and placental index. STZ induced GDM group rats showed the decreased fetuses wight and increased the level of placental weight and placental index. Caffeic acid significantly $(\mathrm{P}<0.001)$ increased the fetuses weight and reduced the placental weight and placental index at dose dependently manner

\section{Effect of caffeic acid on lipid parameters}

Normal control group rats showed the normal pattern of lipid parameters till end of the experimental study. STZ induced GDM rat showed the increased level of TC, TG,
LDL, VLDL and decreased level of HDL and concentration dependent treatment of caffeic acid significantly $(\mathrm{P}<0.001)$ altered the level of lipid parameters (Fig. 4).

\section{Effect of caffeic acid on antioxidant parameters}

Figure 5 showed the effect of caffeic acid of the antioxidant parameters (liver and pancreas) on normal control and STZ induced GDM rats. Normal control group rats showed the almost similar level of antioxidant parameter. STZ induced group rats showed the increased level of MDA and reduced level of SOD, CAT, GPx, GSH in the liver and pancreas as compared to normal control. STZ induced GDM rats received caffeic acid significantly $(\mathrm{P}<0.001)$ reduced the level of MDA and increased the level of SOD, CAT, GPx, GSH in the liver and pancreas. 


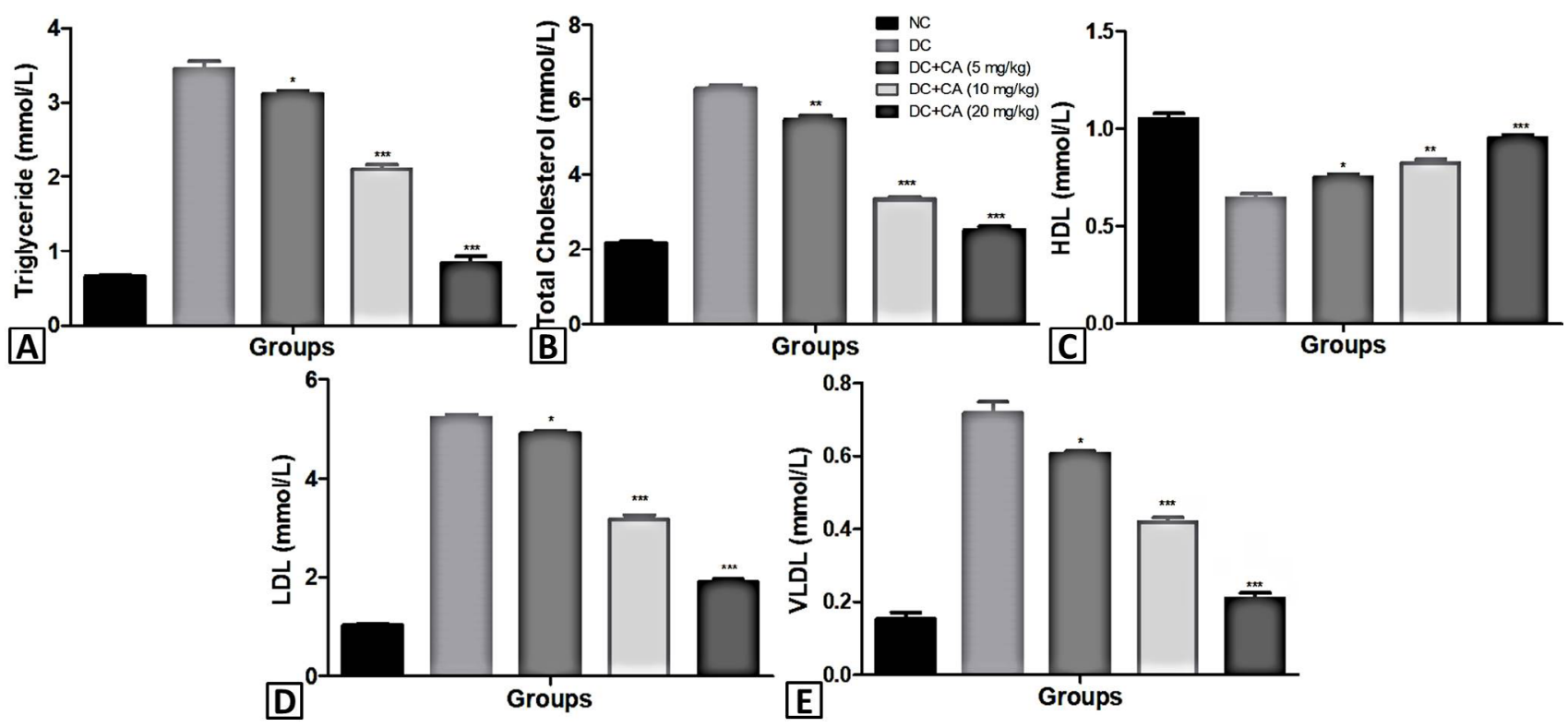

Fig. 4. The effect of caffeic acid on the lipid parameters of streptozotocin (STZ) induced gestational diabetes mellitus (GDM) induced rats at end of the experimental study. A, triglycerides; B, total cholesterol; C, HDL; D, LDL; E, VLDL. Dunnett's multiple test was performed for the statistical analysis. Treated group rats compared with the STZ induced GDM group rats. ${ }^{*} \mathrm{p}<0.05$ consider as the significant, ${ }^{* *} \mathrm{p}<0.01$ consider as the more significant and $* * * \mathrm{p}<0.001$ consider as the most significant.
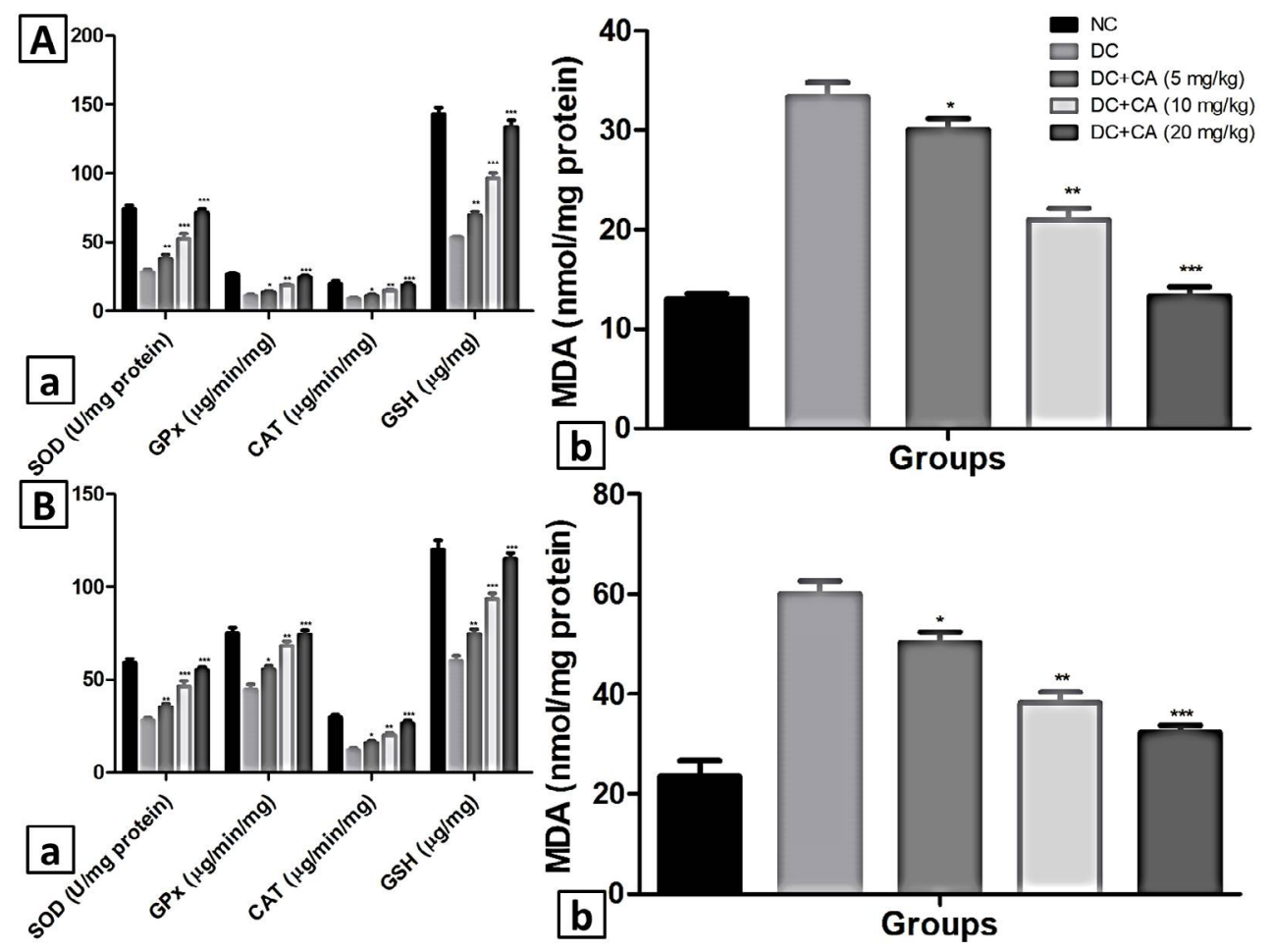

Fig. 5. The effect of caffeic acid on the antioxidant parameter in liver (A) and pancreas (B) of streptozotocin (STZ) induced gestational diabetes mellitus (GDM) induced rats at end of the experimental study. a, SOD, GPx, CAT, GSH; b, MDA. Dunnett's multiple test was performed for the statistical analysis. Treated group rats compared with the STZ induced GDM group rats. ${ }^{*} \mathrm{p}<0.05$ consider as the significant, ${ }^{*} \mathrm{p}<0.01$ consider as the more significant and $* * * \mathrm{p}<0.001$ consider as the most significant. 


\section{DISCUSSION}

Clinical studies suggest that the GDM affects the $10 \%$ of pregnant women and women suffer from the GDM having the higher chance to develop the long-term type 2 diabetes mellitus. The prevalence of GDM is enhancing globally due to recent trend in advance maternal stage and obesity (Shivananjappa and Muralidhara, 2012). Women suffering from the GDM are the high risk to develop the infection and pregnancy complications including ketoacidosis and hypertension. GDM suffer pregnant women on the risk for advance pregnancy complications such as spontaneous abortion, fetal abnormalities, macrosomia, still birth and intrauterine growth restriction (Vafaei-Pour et al., 2018). The frequency of congenital malformations of fetal vital organs is as high as compared to the without complicated pregnancy (Vafaei-Pour et al., 2018). Lot of research conducted on the diabetes, but few of the researcher focus their research only the GDM. However, in the current experimental protocol, we made attempt to estimate the protective effect of caffeic acid on GDM induced via streptozotocin and explore the possible mechanism.

Various researches suggest that the oxidative stress play an important role to develop the cognitive anomalies (Lappas, 2014; Wang et al., 2019). There is enhancing the evidence that showed ROS have a significant role in the pathology of various congenital anomalies such as produced via GDM, cocaine, alcohol intake and radiation (Szmuilowicz et al., 2019). Various teratogenic drugs viz., thalidomide and phenytoin showed the similar mechanism of action. Currently, much work showed that the oxidative stress plays a significant role the diabetes and pathogenesis of diabetic complications (Shivananjappa and Muralidhara, 2012). Clinical and experimental researches have shown that hyperglycemia through various biochemical pathways such as protein glycation and autoxidation leads to boost the formation of ROS (Vafaei-Pour et al., 2018). Similarly, it has been appeared that developing the embryos are very sensitive to high concentration of ROS, particularly during the organogenesis stage.

During the GDM, increased the level of ROS in the mothers due to increase level of protein oxidation, lipid peroxidation (LPO), ribonucleic acid (RNA) and deoxy ribose nucleic acid (DNA) damage in the expansion of embryo (Hong et al., 2017; Yao et al., 2015; Zou et al., 2018). These pathological conditions lead to the more prevalence of skeletal, cardiovascular, brain and other abnormalities in the offspring of diabetic women as compared to the non-diabetic women. In the current experimental study, we observed that the enhanced level of ROS, protein carbonyl and lipid peroxidation and destruction of endogenous enzymes (enzymatic and non enzymatic) antioxidant in the embryo of diabetic rats. Simultaneously, there was a correlation was observed between the degree of oxidative stress and rate of anomalies in embryo (Hong et al., 2017; Yao et al., 2015). Moreover, it has been shown that the controlling the glycaemic control, the rate of diabetic complications remain still high. Still the researcher targeting the diabetes and diabetic complications, but not targeting the oxidative stress induced diabetic complications (Wang et al., 2019). In this current experimental study, we targeting the oxidative stress and its induced the diabetic complication to treat the GDM. In the current experimental study, treatment group reduced the GDM at dose dependent manner due to its antioxidant manner. In the current experimental studies, we observed that the significantly boost the reactive oxygen species (ROS) production in the embryo, protein oxidative and LPO and increased the embryonic antioxidant capacity in the diabetic rats. It is well documented that during the hyperglycemia, increase the generation of free radical in the body and various researches suggest that the degradation of glycated proteins, glucose oxidation and non-enzymatic glycation are responsible for the production of free radicals. During the production of free radicals, endogenous antioxidant such as CAT, SOD, GPx and some vitamins play a significant role to scavenge the free radicals and prevents the side and toxic effects (Lappas, 2014; Wang et al., 2019). Previous investigation suggests that the expansion of embryos is very sensitive to enhance the ROS level, especially during the organogenesis stage. CAT and SOD are the first line endogenous antioxidant enzymes and take part important role in the reduction of ROS in the body (Hong et al., 2017; Yao et al., 2015; Zou et al., 2018). The first line endogenous antioxidant scavenges the free radical, $1 / 2 \mathrm{O}_{2}$, and $\mathrm{OH}$ and reduced the oxidative stress. During the GDM induced a similar result was obtained and dose dependent treatment of caffeic acid reduced the free radical formation via increased the level of endogenous antioxidant. Studies suggest that the ROS is the $2^{\text {nd }}$ messenger and its take part to generate the various redox sensitive signaling pathways (Hong et al., 2017; Zou et al., 2018). The most importantly, its induces the phosphorylation of insulin receptor and substrate in the insulin signalling pathways, subsequently leading to reduced the activity of signal molecules and deteriorate the insulin sensitizing effect and induce the insulin resistance. In the current protocol, we observed that the caffeic acid considerably removed the ROS effect via 2 mechanisms, it could be increase the level of endogenous antioxidant and boost the insulin sensitizing effect and decrease the insulin resistance (Hong et al., 2017; Zou et al., 2018). This effect may be generated due to decrease the blood glucose level 
in the experimental rats and increase the level of insulin.

\section{CONCLUSION}

In summary, the caffeic acid is a powerful antioxidative substance, during the GDM, the excessive generation of free radical and oxidative stress and due to the nature of antioxidative, caffeic acid considerably inhibited the free radical production and oxidative stress. In future, we scrutinized the possible mechanism of action.

\section{Statement of conflict of interest}

The authors declare no conflict of interest.

\section{REFERENCES}

Alfadhli, E.M., 2015. Gestational diabetes mellitus. Saudi med. J., 36: 399-406. https://doi. org/10.15537/smj.2015.4.10307

Bain, E., Crane, M., Tieu, J., Han, S., Crowther, C.A. and Middleton, P., 2015. Diet and exercise interventions for preventing gestational diabetes mellitus. Cochrane Database Syst. Rev., 12: CD010443. https://doi.org/10.1002/14651858. CD010443.pub2

Chen, J.H. and Ho, C.T., 1997. Antioxidant activities of caffeic acid and its related hydroxycinnamic acid compounds. J. Agric. Fd. Chem., 45: 2374-2378. https://doi.org/10.1021/jf970055t

Coustan, D.R., 2013. Gestational diabetes mellitus. Clin. Chem., 59: 1310-1321. https://doi.org/10.1373/ clinchem.2013.203331

de Souza, F.S. and Spinelli, A., 2009. Caffeic acid as a green corrosion inhibitor for mild steel. Corros. Sci., 24: 642-649. https://doi.org/10.1016/j. corsci.2008.12.013

Hong, B., Ding, X., Jia, H. and Zhang, J., 2017. Resveratrol ameliorated gestational diabetes through regulation of AMPK-mediated NF- $\kappa \mathrm{B}$ signaling pathway. Biomed. Res., 28: 3433-3439.

Kumar, V., Ahmed, D., Gupta, P.S., Anwar, F. and Mujeeb, M., 2013a. Anti-diabetic, anti-oxidant and anti-hyperlipidemic activities of Melastoma malabathricum Linn. leaves in streptozotocin induced diabetic rats. BMC Complement. Altern. Med., 13: 222. https://doi.org/10.1186/1472-688213-222

Kumar, V., Ahmed, D., Verma, A., Anwar, F., Ali, M. and Mujeeb, M., 2013b. Umbelliferone $\beta$-Dgalactopyranoside from Aegle marmelos (L.) corr. An ethnomedicinal plant with antidiabetic, antihyperlipidemic and antioxidative activity. $B M C$
Complement. Altern. Med., 13: 273. https://doi. org/10.1186/1472-6882-13-273

Lappas, M., 2014. Activation of inflammasomes in adipose tissue of women with gestational diabetes. Mol. Cell. Endocrinol., 382: 74-83. https://doi. org/10.1016/j.mce.2013.09.011

Lefkovits, Y.R., Stewart, Z.A. and Murphy, H.R., 2019. Gestational diabetes. Medicine, 47: 114-118. https://doi.org/10.1016/j.mpmed.2018.11.006

Maresh, M., 2005. Screening for gestational diabetes mellitus. Semin. Fetal Neonatal Med., 10: 317-323. https://doi.org/10.1016/j.siny.2005.04.009

Maritim, A.C., Sanders, R.A. and Watkins, J.B., 2003. Diabetes, oxidative stress, and antioxidants: A review. J. Biochem. mol. Toxicol., 17: 24-38. https://doi.org/10.1002/jbt.10058

Peyrat-Maillard, M.N., Cuvelier, M.E. and Berset, C., 2003. Antioxidant activity of phenolic compounds in 2,2'-azobis (2-amidinopropane) dihydrochloride (AAPH)-induced oxidation: Synergistic and antagonistic effects. J. Am. Oil chem. Soc., 80: 1007. https://doi.org/10.1007/s11746-003-0812-z

Rajendra-Prasad, N., Karthikeyan, A., Karthikeyan, S. and Venkata-Reddy, B., 2011. Inhibitory effect of caffeic acid on cancer cell proliferation by oxidative mechanism in human HT-1080 fibrosarcoma cell line. Mol. Cell. Biochem., 349: 11-19. https://doi. org/10.1007/s11010-010-0655-7

Sato, Y., Itagaki, S., Kurokawa, T., Ogura, J., Kobayashi, M., Hirano, T., Sugawara, M. and Iseki, K., 2011. In vitro and in vivo antioxidant properties of chlorogenic acid and caffeic acid. Int. J. Pharmaceut., 403: 136-138. https://doi. org/10.1016/j.ijpharm.2010.09.035

Shivananjappa, M. and Muralidhara, M., 2012. Taurine attenuates maternal and embryonic oxidative stress in a streptozotocin-diabetic rat model. Reprod. Biomed. Online, 24: 558-566. https://doi. org/10.1016/j.rbmo.2012.01.016

Spaight, C., Gross, J., Horsch, A. and Puder, J.J., 2016. Gestational diabetes mellitus. Endocr. Dev., 31: 163-178. https://doi.org/10.1159/000439413

Sul, D., Kim, H.S., Lee, D., Joo, S.S., Hwang, K.W. and Park, S.Y., 2009. Protective effect of caffeic acid against beta-amyloid-induced neurotoxicity by the inhibition of calcium influx and tau phosphorylation. Life Sci., 84: 257-262. https://doi. org/10.1016/j.1fs.2008.12.001

Szmuilowicz, E.D., Josefson, J.L. and Metzger, B.E., 2019. Gestational diabetes mellitus. Endocrinol. Metab. Clin. N. Am., 48: 479-493. https://doi. org/10.1016/j.ecl.2019.05.001 
Tang, X., Qin, Q., Xie, X. and He, P., 2014. Protective effect of sRAGE on fetal development in pregnant rats with gestational diabetes mellitus. Cell Biochem. Biophys., 71: 549-556. https://doi. org/10.1007/s12013-014-0233-9

Vafaei-Pour, Z., Shokrzadeh, M., Jahani, M. and Shaki, F., 2018. Embryo-protective effects of cerium oxide nanoparticles against gestational diabetes in mice. Iran. J. pharmceut. Res., 17: 964-975.

Wang, Y., Wang, L., Xu, G. and Wei, D., 2019. Hesperidin exerts the gestational diabetes mellitus via AGEsRAGE signalling pathway. Int. J. Pharmacol., 15:
604-615. https://doi.org/10.3923/ijp.2019.604.615 Yao, L., Wan, J., Li, H., Ding, J., Wang, Y., Wang, X. and Li, M., 2015. Resveratrol relieves gestational diabetes mellitus in mice through activating AMPK. Reprod. Biol. Endocrinol., 13: 118. https:// doi.org/10.1186/s12958-015-0114-0

Zou, C., Zhang, Q. and Zhang, S., 2018. Mogroside IIIE attenuates gestational diabetes mellitus through activating of AMPK signaling pathway in mice. J. pharmacol. Sci., 138: 161-166. https://doi. org/10.1016/j.jphs.2018.09.008 